\title{
EPR Study of UV-irradiated Thymidine Microcrystals Supports Radical Intermediates in Spore Photoproduct Formation
}

\author{
Ellen C. Hayes ${ }^{\dagger}$, Yajun Jian ${ }^{\ddagger}$, Lei Li $i^{\sharp}$, Stefan Stoll ${ }^{* *}$ \\ † Department of Chemistry, Box 351700, University of Washington, Seattle, WA 98195 \\ $¥$ Department of Chemistry \& Chemical Biology, 402 N. Blackford Street, LD 326, Indiana University - \\ Purdue University Indianapolis, Indianapolis, IN 46202
}

\begin{abstract}
Spore photoproduct is a thymidine dimer formed when bacterial endospore DNA is exposed to ultraviolet (UV) radiation. The mechanism of formation of this thymidine dimer has been proposed to proceed through a radicalpair intermediate. The intermediate forms when a methyl-group hydrogen atom of one thymidine nucleobase is transferred to the $\mathrm{C} 6$ position of an adjacent thymidine nucleobase, forming two species, the $\mathrm{TCH}_{2}$ and $\mathrm{TH}$ radicals, respectively. Using a series of thymidine isotopologues and electron paramagnetic resonance (EPR) spectroscopy, we show that microcrystals of thymidine exposed to UV radiation produce these two radical species. We observe three sources which donate the additional hydrogen at the C6 position of the TH radical. One of the three sources is the methyl group of another thymidine molecule in a significant fraction of the $\mathrm{TH}$ species. This lends support to the radical pair intermediate proposed in the formation of spore photoproduct.
\end{abstract}

\section{Introduction}

The spore photoproduct (SP) is a unique DNA lesion formed when two adjacent thymidine bases dimerize upon exposure to UV radiation. Although the pyrimidine bases have been observed to form different classes of dimers, including cyclobutane pyrimidine dimers and pyrimidine 6-4 pyrimidone photoproducts, ${ }^{1}$ the SP, or 5-thyminyl-5,6-dihydrothymine (see Scheme 1) is the dominant photoproduct in bacterial endospore DNA., ${ }^{2,3}$ A combination of the low hydration level of DNA, DNA saturation by small acid-soluble proteins, and a high calcium dipicolinate concentration in dormant endospores creates an environment that favors the A conformation of DNA and formation of the $5 R$ enantiomer (due to DNA helical structure) of SP over other thymidine photoproducts. ${ }^{4-6}$ During spore germination, the $5 R$-SP is efficiently repaired by the $4 \mathrm{Fe}-4 \mathrm{~S}$ cluster enzyme spore photoproduct lyase..$^{7-13}$ The unique structure of SP and its efficient repair make bacterial endospores resistant to the deleterious effects of UV light on DNA for extremely long periods of time. $^{14,15}$ Given the utilization of UV light in sterilization processes, ${ }^{16-18}$ it is important to fully understand the mechanisms of formation and repair of the spore photoproduct.

A long history of progress has led to the current understanding of the dimerization mechanism which forms SP. Originally, a sequential mechanism was suggested that involved the recombination of two thymine radicals; the oxidation product (an allylic 7-yl radical abbreviated $\mathrm{TCH}_{2}$ ) and the reduction product (a 5,6-dihydro-thymid-5-yl radical abbreviated TH). ${ }^{19,20}$ These radicals had been previously observed by continuous-wave electron paramagnetic resonance (EPR) spectroscopy in crystals of thymidine and DNA strands irradiated with gamma and UV radiation. ${ }^{21,22}$
These radicals share the structure of the components of the SP dimer (Scheme 1) making them likely intermediates during dimerization. However, the source of the additional C6 hydrogen of the $\mathrm{TH}$ component was unclear. ${ }^{23}$

To resolve this, Cadet and co-workers irradiated thymidine- $d_{3}$ (deuterated at the nucleobase methyl group) in an ice matrix with UV light and found $60 \%$ incorporation of deuterium at the $\mathrm{C} 6$ position of the $\mathrm{TH}$ moiety of the final product SP (highlighted in red in Scheme 1). ${ }^{5,24}$ The incomplete incorporation of deuterium at the $\mathrm{C} 6$ position was puzzling until recently when Lin et al. performed similar UV irradiation experiments on thin films of methyl deuterated dinucleotide thymine dinucleoside monophosphate $\left(d_{4}-\right.$ TpT or $\left.d_{3}-\mathrm{TpT}\right)$. Upon analyzing the structure of the photoproducts, they discovered the $5 R-\mathrm{SP}$ with complete deuterium incorporation in a stereoselective manner from the methyl group of the 3 ' thymidine to the $\mathrm{C} 6$ position of the $5^{\prime}$ thymidine. ${ }^{25}$ Further studies revealed a substantial kinetic isotope effect (KIE) in SP formation, suggesting that trace unlabeled thymidine$d_{3}$ was the reason for the previously observed incomplete deuterium incorporation at $\mathrm{C}^{2}{ }^{26} \mathrm{~A}$ more detailed mechanism was suggested based on these observations. As depicted in Scheme 1, the $\mathrm{H}$ atom transfer from the methyl group of the 3' thymidine to the $\mathrm{C} 6$ position of the $5^{\prime}$ thymidine is postulated to form an intermediate $\mathrm{TCH}_{2} / \mathrm{TH}$ radical pair which then recombines to the SP. Despite the establishment of the mechanism, these radicals have not yet been directly observed in the SP photochemistry.

This is the author's manuscript of the article published in final edited form as: 


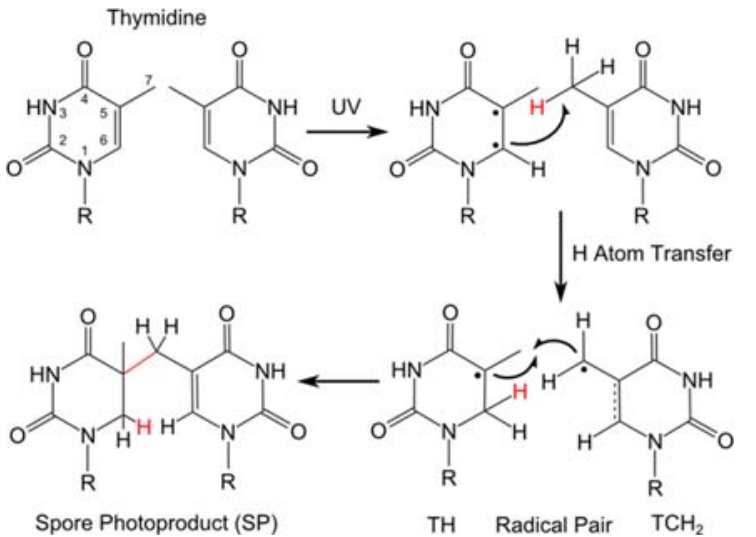

Scheme 1: Proposed mechanism for spore photoproduct formation.

In samples of methyl-deuterated mononucleotide thymidine, Ames et al. showed that 5S-SP forms through the same $\mathrm{H}$ atom transfer mechanism as the biologically relevant $5 R$-SP. ${ }^{26}$ Jian et al. further showed that dry microcrystals of thymidine monomers, in which the thymine bases stack in a left-handed manner, form the 5S-SP as the dominant photoproduct in high yields $(\approx 85 \%)$ upon exposure to UV radiation at room temperature. $^{27,28}$ The base stacking conformation in microcrystals is believed to mimic that found in spore DNA even though these two systems exhibit an opposite chirality (left-handed yielding $5 S$-SP versus right-handed yielding $5 R$-SP, respectively). Considering the purity and high product yield of the $5 S$ SP in thymidine microcrystals exposed to UV radiation, we used this sample preparation in the studies described here to search for the suggested radical intermediates in the SP formation mechanism in Scheme 1.

We used electron paramagnetic resonance (EPR) spectroscopy and isotopically labeled thymidine to probe the radical nature of the proposed intermediates in SP formation. Upon exposing samples of dry microcrystalline powders of thymidine to UV radiation, we observed EPR spectra characteristic of the $\mathrm{TCH}_{2}$ and $\mathrm{TH}$ radicals. Previous studies have thoroughly characterized the structure of the two radical intermediates we observed. ${ }^{21,29-31}$ However, here we use sample preparations known to cleanly form the $5 S$ $\mathrm{SP}^{28}$ and an extensive set of thymidine isotopologues. Site-specific labeling allowed us to identify three sources of the hydrogen at the $\mathrm{C} 6$ position of the $\mathrm{TH}$ radical: an intermolecular methyl group (on the proposed SP pathway), exchangeable hydrogens (off the proposed pathway), and an ambiguous source we term "other" (possibly off the proposed pathway). Applying UV radiation over a wide range of temperatures $(120 \mathrm{~K}, 210 \mathrm{~K}$, and $250 \mathrm{~K})$ results in only a small variation in the ratio of these sources. The observation of $\mathrm{TH}$ radicals formed by $\mathrm{C} 6$ hydrogen addition from the methyl group of another thymidine molecule lends support to the mechanism of SP formation involving the $\mathrm{TCH}_{2} / \mathrm{TH}$ radical pair intermediate.

Table 1: TH radical hyperfine coupling and $g$ values ${ }^{a}$

\begin{tabular}{|c|c|c|c|c|c|}
\hline $\begin{array}{l}\text { starting } \\
\text { material }\end{array}$ & $\begin{array}{l}\text { ionization } \\
\text { conditions }\end{array}$ & $g$ & ${ }^{1} \mathrm{H}_{\mathrm{C} 6}$ & ${ }^{1} \mathrm{H}_{\mathrm{C} 6}$ & $\begin{array}{l}{ }^{1} \mathrm{H}_{\mathrm{C} 7} \\
(3 \mathrm{x})\end{array}$ \\
\hline \multirow{3}{*}{ thymidine $^{21}$} & & 2.0024 & 113.4 & 113.4 & 57.4 \\
\hline & & 2.0030 & 113.4 & 113.4 & 57.4 \\
\hline & & 2.0042 & 113.4 & 113.4 & 57.4 \\
\hline \multirow{3}{*}{ thymidine $\mathrm{e}^{30}$} & \multirow{3}{*}{$\begin{array}{c}\text { X-ray } \\
\text { RT }\end{array}$} & & 123.2 & 117.8 & \\
\hline & & & 113.6 & 107.4 & -- \\
\hline & & & 111.6 & 106 & \\
\hline \multirow{3}{*}{ thymidine $^{32}$} & \multirow{3}{*}{$\begin{array}{c}\text { X-ray } \\
10 \mathrm{~K}\end{array}$} & \multirow{3}{*}{--} & 123.8 & 118.3 & \multirow{3}{*}{--} \\
\hline & & & 114.6 & 107.7 & \\
\hline & & & 112.5 & 105.9 & \\
\hline \multirow{3}{*}{ thymine $\mathrm{e}^{33}$} & \multirow{3}{*}{$\begin{array}{l}\text { X-ray } \\
10 \mathrm{~K}\end{array}$} & \multirow{3}{*}{2.0023} & 133.9 & 97 & 61.3 \\
\hline & & & 124.4 & 86.5 & 53.7 \\
\hline & & & 122.3 & 85.2 & 53.3 \\
\hline \multirow{3}{*}{ thymidine $^{\mathrm{b}, \mathrm{c}}$} & \multirow{3}{*}{$\begin{array}{c}\text { UV } \\
120 \mathrm{~K}\end{array}$} & 2.0021 & $114(10)$ & $110(10)$ & $54(5)$ \\
\hline & & 2.0067 & $114(10)$ & $110(10)$ & $56(5)$ \\
\hline & & 2.0080 & $117(10)$ & $113(10)$ & $64(5)$ \\
\hline
\end{tabular}

${ }^{a}$ Hyperfine coupling principal values are in $\mathrm{MHz}$

${ }^{\mathrm{b}}$ Simulation parameters from this study

${ }^{\mathrm{c}}$ Error in $\mathrm{g}$ values is \pm 0.003 (due to $0.5 \mathrm{mT}$ magnet error); error in hyperfine coupling based on varying simulations

Table 2: $\mathrm{TCH}_{2}$ radical hyperfine coupling and $\mathrm{g}$ values ${ }^{\mathrm{a}}$

\begin{tabular}{|c|c|c|c|c|c|c|}
\hline $\begin{array}{l}\text { starting } \\
\text { material }\end{array}$ & $\begin{array}{l}\text { ionization } \\
\text { conditions }\end{array}$ & $g$ & ${ }^{1} \mathrm{H}_{\mathrm{C} 7}$ & ${ }^{1} \mathrm{H}_{\mathrm{C} 7}$ & ${ }^{1} \mathrm{H}_{\mathrm{C} 6}$ & ${ }^{14} \mathrm{~N} 1$ \\
\hline thymidine $^{31}$ & $\begin{array}{c}\text { X-ray } \\
\text { RT }\end{array}$ & -- & $\begin{array}{c}65.8 \\
42.8 \\
21\end{array}$ & $\begin{array}{c}67 \\
42.5 \\
22\end{array}$ & $\begin{array}{l}42.2 \\
29.7 \\
14.3\end{array}$ & -- \\
\hline thymidine $^{32}$ & $\begin{array}{c}\text { X-ray } \\
10 \mathrm{~K}\end{array}$ & -- & $\begin{array}{l}-70.2 \\
-42.8 \\
-22.2\end{array}$ & $\begin{array}{l}-65.4 \\
-42.9 \\
-20.4\end{array}$ & $\begin{array}{c}-41.8 \\
-29.7 \\
-14\end{array}$ & -- \\
\hline $\begin{array}{l}\text { 1-methyl- } \\
\text { thymine }^{32}\end{array}$ & $\begin{array}{c}\text { X-ray } \\
10 \mathrm{~K}\end{array}$ & -- & $\begin{array}{l}-72.5 \\
-44.1 \\
-23.1\end{array}$ & $\begin{array}{c}-67.7 \\
-43.6 \\
-21\end{array}$ & $\begin{array}{l}-37.4 \\
-27.1 \\
-13.1\end{array}$ & -- \\
\hline thymine ${ }^{33}$ & $\begin{array}{c}\text { X-ray } \\
10 \mathrm{~K}\end{array}$ & 2.0023 & $\begin{array}{c}-66.6 \\
-43.2 \\
-22\end{array}$ & $\begin{array}{l}-71.1 \\
-44.3 \\
-22.3\end{array}$ & $\begin{array}{l}-43.5 \\
-31.2 \\
-15.1\end{array}$ & $\begin{array}{c}-5.2 \\
-4.9 \\
2\end{array}$ \\
\hline thymidine $^{34}$ & $\begin{array}{l}\text { UV } \\
77 \mathrm{~K}\end{array}$ & -- & $\begin{array}{c}64.4 \\
42 \\
19.6\end{array}$ & $\begin{array}{c}64.4 \\
42 \\
19.6\end{array}$ & $\begin{array}{c}39.2 \\
28 \\
14\end{array}$ & -- \\
\hline thymidine $^{\mathrm{b}, \mathrm{c}}$ & $\begin{array}{c}\text { UV } \\
120 \mathrm{~K}\end{array}$ & $\begin{array}{l}2.0045 \\
2.0055 \\
2.0065\end{array}$ & $\begin{array}{r}-56(5) \\
-42(5) \\
-19(5)\end{array}$ & $\begin{array}{l}-56(5) \\
-42(5) \\
-22(5)\end{array}$ & $\begin{array}{r}-36(5) \\
-28(5) \\
-20(5)\end{array}$ & $\begin{array}{r}-1(5) \\
-1(5) \\
5(5)\end{array}$ \\
\hline
\end{tabular}

${ }^{\text {a }}$ Hyperfine coupling principal values are in $\mathrm{MHz}$

${ }^{\mathrm{b}}$ Simulation parameters from this study

${ }^{\mathrm{c}}$ Error in $\mathrm{g}$ values is \pm 0.003 (due to $0.5 \mathrm{mT}$ magnet error); error in hyperfine coupling based on varying simulations 


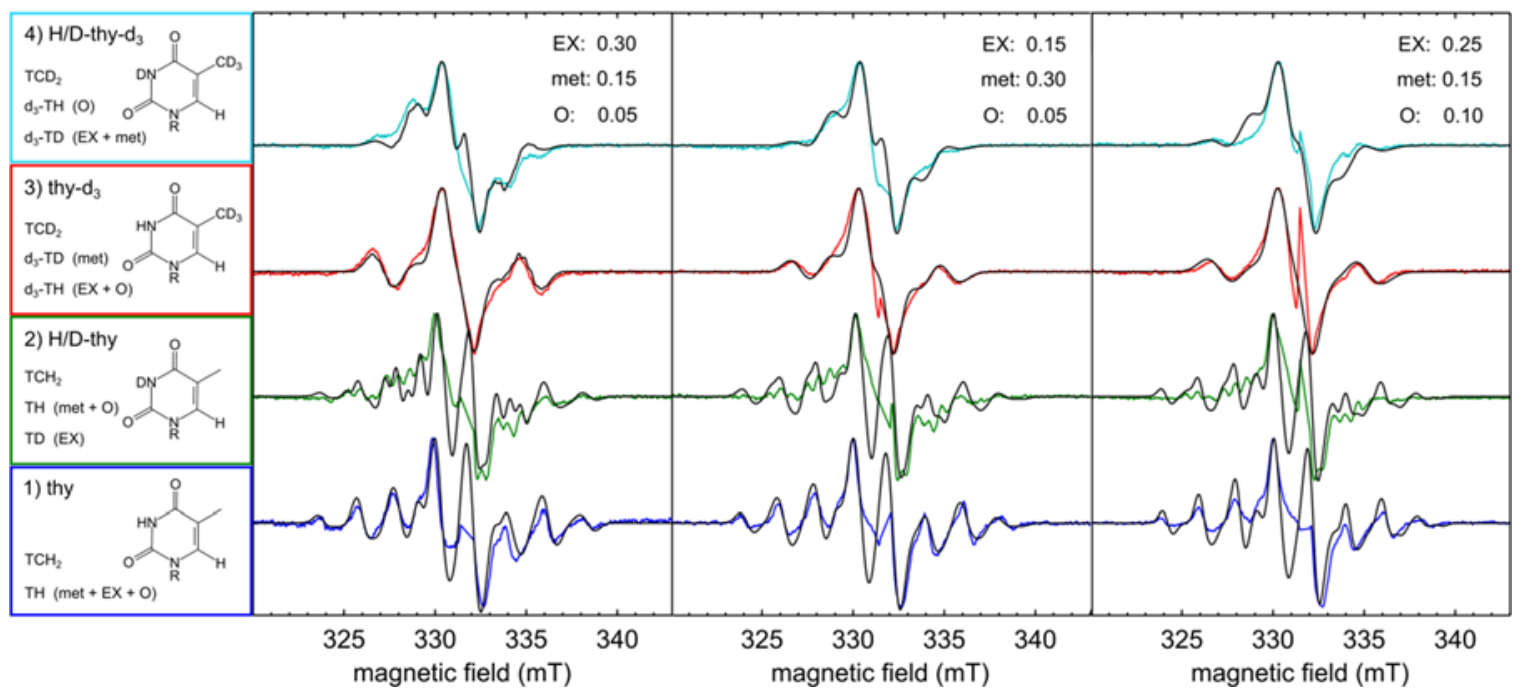

Figure 1. Continuous-wave EPR spectra of microcrystalline powder samples of thymidine (blue), H/D-thymidine (green), thymidine- $d_{3}$ (red), and H/D-thymidine- $d_{3}$ (cyan) photodamaged with $266 \mathrm{~nm}$ laser pulses at $120 \mathrm{~K}, 210$ $\mathrm{K}$, and $250 \mathrm{~K}$ indicated at the top of each set of axes. In all cases, EPR measurements were carried out at $120 \mathrm{~K}$. The structures of the starting materials are shown in the left column. The isotopologues of the $\mathrm{TCH}_{2}$ and $\mathrm{TH}$ radicals that are used to simulate the spectra are indicated to the left of each starting material. In addition, the hydrogen sources (met, EX, or O defined in main text) that lead to particular TH isotopologues for each starting material are indicated in parenthesis. Simulations were done assuming the restrictive model (defined in main text) and the fractions of hydrogen sources producing TH isotopologues at a given temperature of UV irradiation are indicated at the top right corner of each panel. Simulated spectra are shown in black. EPR spectrometer settings were as follows: microwave power of $0.1 \mathrm{~mW}$ to avoid power saturation; microwave frequency between 9.297$9.304 \mathrm{GHz}$ (simulations take in to account the frequency variation between samples); typically 30-100 scans were needed to achieve the signal-to-noise ratio of the spectra shown. In all cases the spectra were normalized to their most intense feature for clarity. At the center of each experimental spectrum was a feature attributed to a quartzbased radical which was removed for clarity.

\section{Results}

Selective deuterium labeling of thymidine can be used to determine the source of the additional hydrogen at the $\mathrm{C} 6$ position of the proposed $\mathrm{TH}$ radical intermediate. Deuterium has a nuclear spin of 1 , but due to its decreased gyromagnetic ratio, has hyperfine coupling strengths $\approx 6.5$ times smaller than protium. The mechanism of spore photoproduct formation suggested in Scheme 1 indicates the additional hydrogen at the $\mathrm{C} 6$ position of the TH radical will come from the methyl group of an adjacent thymidine (which becomes the $\mathrm{TCH}_{2}$ radical). However, other possible off-pathway sources of the additional hydrogen at C6 of TH are the exchangeable hydrogens of thymidine at $\mathrm{N} 3$ of the base and O3' and O5' of the sugar. Here we use four isotopologues of thymidine to distinguish these hydrogen sources; "thy" is the fully protiated thymidine, "H/D-thy" is thymidine deuterated at the positions with exchangeable hydrogens, "thy- $d_{3}$ " is thymidine deuterated at the C5 methyl group, and "H/D-thy- $d_{3}$ " is thymidine deuterated at the C5 methyl group and at positions with exchangeable hydrogens. The EPR spectra resulting from UV irradiation (266 $\mathrm{nm}, 4.66 \mathrm{eV}$ ) of microcrystals of these four thymidine isotopologues at $120 \mathrm{~K}, 210 \mathrm{~K}$, and $250 \mathrm{~K}$ are shown in Figure 1. In all cases, after exposure to UV radiation at the temperatures indicated in the figure, the EPR spectra were recorded at $120 \mathrm{~K}$ to increase the signalto-noise ratio.

With our choices in isotopologues of thymidine starting material we can distinguish three sources of the additional hydrogen at $\mathrm{C} 6$ of the $\mathrm{TH}$ radical; the methyl group (abbreviated "met", on proposed SP formation pathway), the exchangeable hydrogens (abbreviated "EX", off proposed SP pathway), or any other hydrogens in the system (abbreviated "O", possibly off proposed SP pathway). In total, only two spectroscopically distinct isotopologues of the $\mathrm{TCH}_{2}$ radical $\left(\mathrm{TCH}_{2}\right.$ and $\left.\mathrm{TCD}_{2}\right)$ and four isotopologues of the $\mathrm{TH}$ radical $\left(\mathrm{TH}, \mathrm{TD}, d_{3}-\mathrm{TH}\right.$, and $d_{3}-\mathrm{TD}$, structures in Figure 2) can form. The starting materials will produce predictable isotopologues of the $\mathrm{TH}$ radicals depending on the source of the additional hydrogen at C6. In the 
left column of boxes in Figure 1, we summarize which $\mathrm{TCH}_{2}$ and $\mathrm{TH}$ isotopologues will form in a given starting material. In addition, the source of the additional C6 hydrogen (either met, EX, or O) which forms the TH isotopologues is indicated in parentheses.

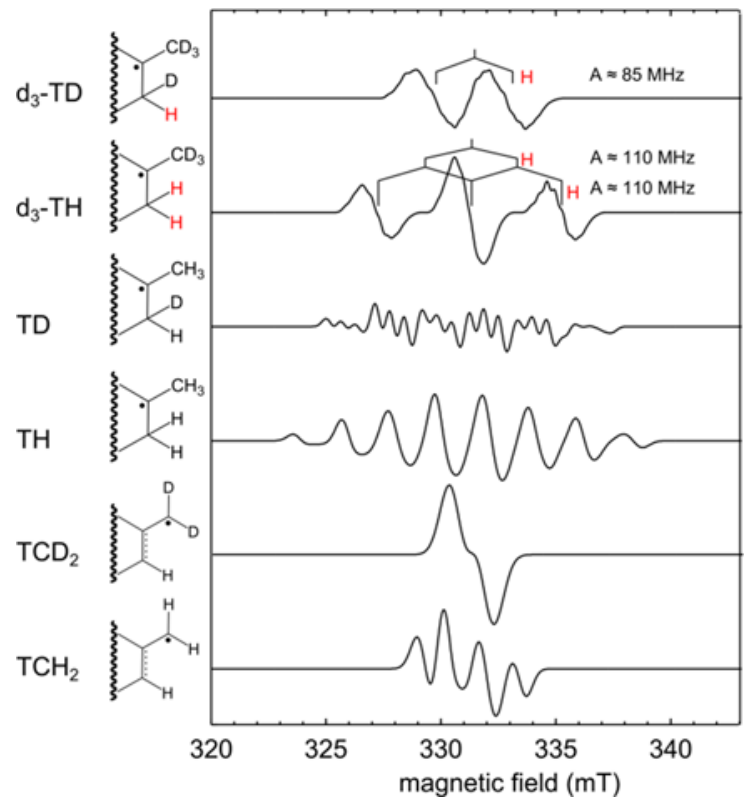

Figure 2. Simulations of the $9.3 \mathrm{GHz}$ EPR spectra of the six radicals (two $\mathrm{TCH}_{2}$ isotopologues, four $\mathrm{TH}$ isotopologues) that appear as components in the mixtures that give the spectra of Figure 1. Simulated spectra are area normalized.

In order to gain an understanding of the magnetic properties of the suggested radical intermediates in SP formation we performed density functional theory (DFT, B3LYP, 6-311G) calculations. For $\mathrm{TCH}_{2}$, DFT calculations reveal substantial spin density on $\mathrm{C} 5, \mathrm{C} 6$, and $\mathrm{C} 7$ which results in strong hyperfine coupling to the protons at positions 6 and 7. For TH, DFT calculations show substantial spin density on $\mathrm{C} 5$ which results in hyperfine coupling to the three methyl protons and very strong couplings to the two protons at the $\mathrm{C} 6$ position (Figure 3 and Tables 1 and 2).

To begin spectral simulations, the bottom spectrum in Figure 1A (thy starting material) was simulated as a 1:1 ratio of the $\mathrm{TCH}_{2}$ and $\mathrm{TH}$ radicals using the results of DFT calculations for EPR properties of the $\mathrm{TCH}_{2}$ and $\mathrm{TH}$ radicals. Then the principal values of the magnetic parameters were varied until adequate agreement between the simulated and experimental spectra was achieved. This produced the magnetic property values of the primary isotopologues which are in reasonable agreement with the literature values in Tables 1 and 2 (also see SI Section 1). To simulate EPR spectra of $\mathrm{TCH}_{2}$ and $\mathrm{TH}$ isotopologues we scaled down the appropriate proton hyperfine coupling values of the primary isotopologues using the ratio of gyromagnetic ratios $\gamma_{1_{\mathrm{H}}} / \gamma_{2_{\mathrm{H}}} \approx 6.5$. In the case of the $d_{3}$-TD isotopologue, the hyperfine coupling of the one strongly coupled proton was scaled down to $75 \%$ of the hyperfine coupling of the primary $\mathrm{TH}$ isotopologue. The scaled-down value $\left(A_{\text {iso }} \approx 85 \mathrm{MHz}\right)$ is within the range of $\mathrm{C} 6$ proton couplings reported in the literature (see Table 1). The Fermi contact term of these hyperfine coupling values are expected to depend strongly on the dihedral angle C7-C5-C6-HC6. Structural variation when one deuterium nucleus is present at C6 may be the cause for the decrease. The hyperfine coupling values of all isotopologues are summarized in the supplementary information (Tables $\mathrm{S} 1 \mathrm{a}$ and $\mathrm{S} 1 \mathrm{~b})$.

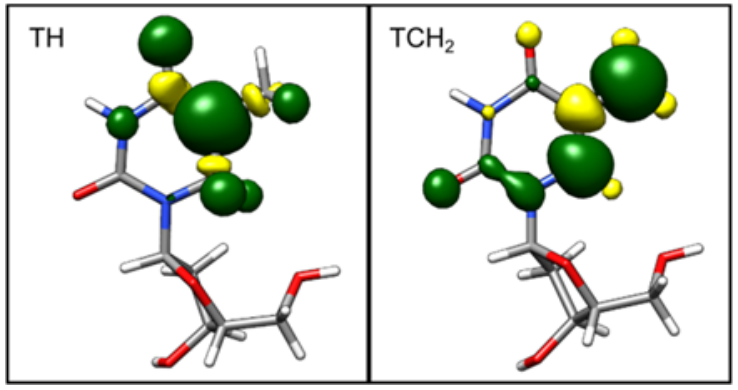

Figure 3. Calculated spin density distributions (isosurface contoured at $\pm 0.0025 a_{0}^{-3}$ ) of the TH and $\mathrm{TCH}_{2}$ radicals. Positive spin density is shown in green and negative spin density is shown in yellow.

For clarity, the spectra of $\mathrm{TCH}_{2}$ and $\mathrm{TH}$ isotopologues are separately simulated in Figure 2 . The $d_{3}$-TH radical has a triplet pattern with features at $\approx 327$ $\mathrm{mT}, \approx 331 \mathrm{mT}$, and $\approx 335 \mathrm{mT}$. This is due to strong coupling, $A$, to two protons at C6. The $d_{3}$-TD spectrum is distinguished by strong hyperfine coupling to one proton at C6. This produces a doublet pattern with features at $330 \mathrm{mT}$ and $333 \mathrm{mT}$. This radical can form in thy- $d_{3}$ only when "met" is the hydrogen source. It is the presence of the $d_{3}$-TD radical in the UVphotodamaged products of thy- $d_{3}$ that will lend support to the mechanism of SP formation in Scheme 1 involving the radical pair intermediate.

It is clear that the experimental spectra in Figure 1 are mixtures of the individual spectra simulated in Figure 2. We developed a "restrictive model" to quantitate these mixtures of $\mathrm{TCH}_{2}$ and $\mathrm{TH}$ isotopologues. To perform simulations, we assumed an equal ratio $(1: 1)$ of the $\mathrm{TCH}_{2}: \mathrm{TH}$ isotopologues in each spectrum to account for the expected stoichiometry of oxidation and reduction (transfer of one electron and hydron) which we call the " $1: 1$ restriction". In addition, we assumed that the ratio of hydrogen sources "met", "EX", and "O" leading to the reduction products (TH isotopologues) was identical for all starting materials at a given UV irradiation temperature. As this assumption essentially excludes kinetic isotope effects, we call it the "isotope effect restriction". These assumptions 
allowed us to simultaneously simulate the four spectra collected at a particular temperature. We found one set of fractions of the three hydrogen sources (which produce predictable $\mathrm{TH}$ isotopologues with respect to the particular starting material) that yielded an adequate simulation for each of the four spectra.

The spectrum for H/D-thy- $d_{3}$ in Figure 1A reveals the percentage of $\mathrm{TH}$ isotopologue which forms due to the "O" hydrogen source $(5 \%)$, as this is the only way to produce the $d_{3}-\mathrm{TH}$ radical in this material. The spectrum for thy- $d_{3}$ in Figure 1A reveals the percentage of TH isotopologues $(15 \%)$ which form due to the "met" hydrogen source, as this is the only way to produce to the $d_{3}$-TD radical from this starting material. Finally, the "EX" hydrogen source was used to account for the remaining $30 \%$ of $\mathrm{TH}$ isotopologues which should be produced assuming the 1:1 restriction. The ratios of hydrogen sources which produce the $\mathrm{TH}$ radicals are summarized at the top right corner of Figure 1A next to the abbreviations "met", "EX", and "O". With the same assumptions and methodology, the ratios of hydrogen sources producing $\mathrm{TH}$ isotopologues at $210 \mathrm{~K}$ and $250 \mathrm{~K}$ were determined and the results listed in the top right corners of Figures $1 \mathrm{~B}$ and $1 \mathrm{C}$.

Despite the limiting assumptions in our model, the agreement between experimental and simulated spectra is satisfactory. However, as there are obvious visual disagreements, we also performed less restrictive simulations. In Figure S1, we show the same data as in Figure 1, simulated without assuming either the isotope effect restriction or the 1:1 restriction. In addition, the hyperfine coupling of the $\mathrm{TH}$ isotopologues were altered slightly from the primary isotopologue $(\mathrm{TH}$ formed from UV irradiation at $120 \mathrm{~K}$ ) but still kept within $25 \%$ of the primary values (parameters in Tables S2a and S2b). Removing these restrictions allows for better visual agreement with the experimental spectra. Generally, the new simulations indicate that the ratio of $\mathrm{TCH}_{2}: \mathrm{TH}$ isotopologues is roughly $1: 1$ after UV irradiation at $120 \mathrm{~K}$, but increases to $2: 1$ after UV irradiation at $250 \mathrm{~K}$. In addition, the relative contribution of $\mathrm{TH}$ radicals produced with the "O" hydrogen source compared to $\mathrm{TH}$ radicals produced by the "met"+ "EX" sources increases at warmer temperatures of UV irradiation. Most importantly, the simulations for thy- $d_{3}$ exposed to UV radiation at all three temperatures must include some contribution from the $d_{3}$-TD radical, indicating a methyl group source for the additional hydrogen at the $\mathrm{C} 6$ position of the TH isotopologues which supports $\mathrm{TH}$ formation through the mechanism shown in Scheme 1.

We explored the reproducibility of our results. In general, for a particular starting material UV irradiated at a given temperature, the identities of radical isotopologues present in the spectra are reproducible (see Figures S2 and S3). However, the ratio of radicals varies. For instance, in Figure S4 we show four different trials of thy- $d_{3}$ irradiated at $120 \mathrm{~K}$ which we simulated using three different schemes. We find the percentage of $d_{3}$-TD varies from $0 \%$ to $25 \%$ among the four sample trials. Although it is tempting to try to draw quantitative conclusions from our simulations, the poor reproducibility as shown in Figure S4 precludes extensive interpretation.

UV radiation was applied at $120 \mathrm{~K}$ to trap higher yields of intermediates. However, since the reaction in spore DNA takes place at biological temperatures, we also applied UV radiation at the warmer temperatures of $210 \mathrm{~K}$ and $250 \mathrm{~K}$. We note the features due to the $d_{3}$ TD radical in thy- $d_{3}$ (evidence for the "met" hydrogen source which supports the suggested SP mechanism) are most prominent in the sample irradiated at $210 \mathrm{~K}$ indicating this may be the best temperature we tested for trapping this intermediate. Applying UV radiation at $250 \mathrm{~K}$ led to decreased signal intensity and spectral broadening; therefore, UV irradiation temperatures above $250 \mathrm{~K}$ were not pursued.

We also explored the temperature dependence of the spectra after completion of the irradiation. This helped to identify the reason for increased broadening in the spectra of samples exposed to UV radiation at temperatures greater than $120 \mathrm{~K}$. The increased line width may be due to two different types of broadening. First, there is the homogeneous broadening that occurs due to spin relaxation which should become more pronounced at warmer temperatures. Indeed, increasing the EPR measurement temperature to $260 \mathrm{~K}$ for material UV irradiated at $120 \mathrm{~K}$ broadens out the hyperfine features (SI FigureS5A). Second, there is site-to-site inhomogeneity in the microcrystalline lattice. There is more thermal energy in starting material exposed to UV radiation at warmer temperatures which may allow the radicals to populate a wider range of conformations as the photodamage occurs. For example, although the features of material $\mathrm{UV}$ irradiated at $210 \mathrm{~K}$ and $250 \mathrm{~K}$ sharpen as the measurement temperature is dropped to $120 \mathrm{~K}$ (decreased homogeneous broadening as stated above, SI Figure S5B and C) the spectra do not reach the sharpness observed for samples exposed to UV radiation at $120 \mathrm{~K}$. Importantly, variation of the EPR measurement temperatures shows no new species are formed when UV is applied at variable temperatures.

Therefore, we also performed measurements on thin film samples (SI Figures S2 an S3), since UV irradiation of thymidine thin films (formed by evaporation of methanol) has also been shown to produce the SP dimer. ${ }^{26}$ The results show the same formation of $\mathrm{TCH}_{2}$ and $\mathrm{TH}$ radical isotopologues, but generally with broadened spectral features compared to the microcrystal samples, supporting the idea that some of the broadening is caused by structural heterogeneity.

\section{Discussion}

Site-selective isotopic labeling has more clearly established the sources of the additional hydrogen at the C6 position of the TH radicals formed when 
microcrystals of thymidine are exposed to UV radiation. We modeled these sources of hydrogen with restrictive spectral simulations of our data in Figure 1. Fits are improved with an unrestrictive model where we relaxed the 1:1 restriction and the isotope effect restriction (Figure S1). In Figure S6, we graphically represent the ratio of isotopologues used in the restrictive and unrestrictive models in Figures 1 and S1. Using the two models and the graphical representation of the results, we understand the underlying reasons for the misfits in Figure 1 and can identify trends in the data. We will now discuss our findings in the context of previous literature concerning both the formation of spore photoproduct and the more general effects of ionizing radiation on thymidine.

\section{Observation of the "met" hydrogen source}

It is not surprising that a mixture of sources ("met", "EX", and "O") donate the additional hydrogen at C6 of the TH isotopologues. Previously, it was observed that crystals of thymidine grown in $\mathrm{D}_{2} \mathrm{O}$ (H/D-thy) formed the TD isotopologue (our "EX" source) when $\mathrm{X}$-ray radiation was applied at $77 \mathrm{~K}$. Alternatively, when UV radiation $(320 \mathrm{~nm})$ was applied to H/D-thy at $77 \mathrm{~K}$, the TH isotopologue (our "met" or "O" source in H/D-thy) was the dominant product. ${ }^{34}$ This latter result is in contrast to our measurements where we see TD as the dominant isotopologue formed in H/D-thy upon UV irradiation at or above $120 \mathrm{~K}$. Although this latter result from the literature is indicative of the "met" source, in these studies isotopic labeling of the methyl group was not performed to confirm the source of the C6 hydrogen in TH. It was observed that dry films of thymidine- $d_{3}$ and DNA labeled with thymine- $d_{3}$ formed the $d_{3}-\mathrm{TH}$ isotopologue when exposed to UV radiation at $77 \mathrm{~K}^{22}$ (analogous to our "EX" and "O" in thy- $d_{3}$ ). The poor resolution of these early experiments did not allow observation of the $d_{3}$-TD radical (our "met" source) if it was present. Our simulations have allowed us to quantitate the contributions of three C6 hydrogen sources which lead to the TH isotopologues. Compared to other studies we observe the "met" source of the additional hydrogen at $\mathrm{C} 6$ of the $\mathrm{TH}$ radical which is indicative of radical intermediates in SP formation.

\section{Relaxing the isotope effect restriction}

Using the unrestrictive model, simulations of the data are improved when the ratios of hydrogen sources are allowed to vary for different thymidine isotopologues at a given temperature of UV radiation. This is likely due to isotope effects. SP formation has a KIE of 3.5 in dinucleotide thymidine (TpT) thin films exposed to UV radiation. ${ }^{25}$ This deuterium discrimination triples for monomeric thymidine thin films. ${ }^{26}$ Considering this, formation of the $\mathrm{TH}$ radical via the SP mechanism ("met") should be more favorable in thymidine with protiated methyl group than in thymidine with deuterated methyl group. In our samples, this would manifest as a larger relative ratio of TH ("met" + "O") in H/D-thy than the ratio of $d_{3}$-TD ("met") in thy- $d_{3}$ for a given temperature of UV irradiation. However, in
Figure S6 (right) we can see the ratio of $d_{3}$-TD in thy$d_{3}$ is larger at all three temperatures. One could speculate that non-deuterated starting material is more likely to proceed to the final product, SP, thus quenching more of the TH radicals in H/D-thy samples. III. Isotope effects: the " $O$ " source of hydrogen

The large deuterium discrimination in SP formation potentially accounts for our "O" source of hydrogen. The "met" and "O" sources may be equivalent with the "O" source resulting from the trace ${ }^{1} \mathrm{H}$ impurities in the synthesis of thymidine- $d_{3}$ (99.5\% pure) ${ }^{26}$ If this is the case, the impurities account for $\approx 25 \%, 14 \%$, and 40 $\%$ of the $\mathrm{TH}$ isotopologues formed by the SP mechanism ("met" or "O") at $120 \mathrm{~K}, 210 \mathrm{~K}$, and $250 \mathrm{~K}$, respectively (using the hydrogen source fractions obtained from our restrictive model in Figure 1). With a $0.5 \%$ impurity, this would indicate 50 -fold, 28 -fold, and 80 -fold deuterium discriminations, respectively. As these numbers are larger than the reported discrimination, there may also be incomplete labeling of the exchangeable positions in the H/D-thy- $d_{3}$ sample which we use to measure the percentage of $\mathrm{TH}$ isotopologues formed by the "O" source of hydrogen.

In addition, there is a larger relative fraction of $\mathrm{TH}$ isotopologues produced by the "O" source at warmer temperatures of UV irradiation using either the restrictive or unrestrictive model. Previous EPR studies have been carried out on thymidine crystals grown in $\mathrm{D}_{2} \mathrm{O}$ (analogous to our starting material H/D-thy). Gamma irradiation of these single crystals at $77 \mathrm{~K}$ produced the TD isotopologue (our "EX" source). ${ }^{35}$ Upon annealing these samples to warmer temperatures, the deuterium began to exchange for protium at room temperature and fully exchanged after annealing to 373 $\mathrm{K}$ forming the $\mathrm{TH}$ isotopologue. Our EPR spectra do not show obvious signs of $\mathrm{H} / \mathrm{D}$ exchange upon warming the samples irradiated at $120 \mathrm{~K}$ to $260 \mathrm{~K}$ (Figure S2). However, the increase in the ratio "O"/"met+EX" ( 0.25 at $120 \mathrm{~K}$ to 0.65 at $250 \mathrm{~K}$, unrestrictive model) in $\mathrm{H} / \mathrm{D}$-thy- $d_{3}$ samples may be indicative of deuterium-to-protium exchange occurring during the photodamage process at warmer temperatures of UV irradiation.

IV. Relaxing the 1:1 restriction

Relaxing the 1:1 restriction in our model also improves the agreement of the simulations with the experimental spectra (compare Figure 1 to Figures S1 and $\mathrm{S} 4$ ). In several previous studies, the $\mathrm{TCH}_{2}$ radical is reported as a minor species in comparison to the $\mathrm{TH}$ radical. ${ }^{21,31,34}$ In addition, the $\mathrm{TCH}_{2}$ radical has been reported to form as a radical pair in gamma irradiated single crystals of thymine where $\mathrm{H}_{2}$ is believed to form in the process. ${ }^{36}$ In this instance, two oxidation products form with no mention of the corresponding reduction product. The graphic in Figure S6 (right) illustrates that the ratio $\mathrm{TCH}_{2}: \mathrm{TH}$ trends from $1: 1$ to $2: 1$ as the UV irradiation temperature is increased in our experiments. This is in contrast to the literature where the $\mathrm{TCH}_{2}$ radical is reported as a minority species in single 
crystals of irradiated thymidine at cryogenic temperatures and at room temperature.

$V$. The "EX" source of hydrogen

Previous literature suggests a possible mechanism for the "EX" source. The $\mathrm{T}^{-}$radical (one electron addition) is a potential precursor to the TH radical (one electron, one proton addition). The $\mathrm{T}^{+}$radical (one electron loss) is a potential precursor to the $\mathrm{TCH}_{2}$ radical (one electron loss, deprotonation at the methyl group). Holroyd and Glass observed the TH and TD radicals in glasses of thymidine in $\mathrm{H}_{2} \mathrm{O}$ and $\mathrm{D}_{2} \mathrm{O}$, respectively, which they postulated to form through hydronation of the $\mathrm{T}^{-}$radical. ${ }^{37}$ Further studies by Sevilla established that the $\mathrm{T}^{-}$and $\mathrm{T}^{+}$radical can form in frozen aqueous solutions of thymine exposed to UV radiation $(77 \mathrm{~K}) \cdot{ }^{38-41}$ In these studies, $\mathrm{T}^{+}$and $\mathrm{T}^{-}$were observed to decay to the $\mathrm{TCH}_{2}$ and $\mathrm{TH}$ radicals, respectively, upon increasing the temperature to 143 K. ${ }^{38}$ Hydronation at the $\mathrm{C} 6$ position of the anion radicals of thymine and uracil has also been followed at room temperature by both electronic absorption and solution state EPR spectroscopies. ${ }^{42,43}$ It is possible that our TH isotopologues produced by the "EX" source of hydrogens also form through hydronation of the $\mathrm{T}^{-}$ radical. However, the literature results were obtained for aqueous samples of thymine (not thymidine) exposed to UV radiation. In solvent-free thymidine crystals, the $\mathrm{T}^{-}$radical, along with the $\mathrm{TCH}_{2}$ and $\mathrm{TH}$ radicals, have been observed at irradiation (X-ray) and EPR measurement temperatures of $10 \mathrm{~K}$ and lower. $^{30,32,33,44,45}$ Warming these samples to $40 \mathrm{~K}$ caused decay of the anion radical but did not lead to observable increase in the concentration of $\mathrm{TH}$ radical ${ }^{32,33,45}$ suggesting that in the solid state the anion does not clearly decay into the $\mathrm{TH}$ radical. Our temperature variation studies did not reveal changes in radical species, so we cannot conclude that our "EX" source is equivalent to hydronation of the $\mathrm{T}^{-}$radical (Figures $\mathrm{S} 2$, S3, and S5).

VI. Presence of alternative radicals: $T^{-}$and $T^{+}$

The substantial improvement in simulations we achieve after relaxing our restrictions helps substantiate our assumption that only $\mathrm{TCH}_{2}$ and $\mathrm{TH}$ isotopologues are present in our system. However, as stated, the $\mathrm{T}^{+}$ and $\mathrm{T}^{-}$radicals are relevant species in solution and the $\mathrm{T}^{-}$radical (SI Figure S7) is a relevant product in the irradiation of DNA strands. ${ }^{46,47}$ Given the simplicity of the $\mathrm{T}^{-}$EPR spectrum (SI Figure S7) we cannot completely rule out its presence in our spectra, but we can adequately simulate our data without it. The $\mathrm{T}^{+}$ radical would produce a more distinct spectrum (SI Figure S7) so it seems unlikely that it is present in our spectra. In a more complicated situation, Malone et al. irradiated thymidine monophosphate at $77 \mathrm{~K}$ in aqueous perchlorate glass and found a mixture of $\mathrm{TCH}_{2}, \mathrm{TH}$, and anion radicals. Warming this sample to $240 \mathrm{~K}$ caused decay of the $\mathrm{TH}$ radical with no observable successor and decay of the $\mathrm{TCH}_{2}$ radical to a quintet species. ${ }^{48}$ Despite these observations in other systems, we do not need to include additional radical species to achieve better simulations of our data.

VII. Lack of expected radical pair interaction

We do not see evidence in our spectra for the strong electron-electron interaction expected for the radical pair intermediate directly on the proposed pathway to $\mathrm{SP}$ in Scheme 1. Previously, Dulčić et al. observed very-low-intensity signals assigned to pairs of $\mathrm{TCH}_{2}$ radicals $\approx 5 \AA$ apart in thymidine crystals with dipolar splitting on the order of $\approx 15 \mathrm{mT} .{ }^{36}$ Based on the crystal structure by Young et al., ${ }^{27}$ the atoms of adjacent thymidine rings are $\approx 3-4 \AA$ apart which would result in a dipolar splitting of 30-60 mT for a radical pair in our microcrystals. Our spectra are adequately simulated without any electron-electron coupling. Our simulations utilize line broadenings less than about 1 $\mathrm{mT}$ which corresponds to radicals at least $12 \AA$ apart. Previous studies $^{25}$ and theoretical calculations ${ }^{49}$ of the SP formation mechanism also suggest that an excited triplet state forms prior to the rate-limiting $\mathrm{H}$-atom abstraction step which forms the radical pair. Here, we saw no evidence of a triplet state (no half field signal, data not shown).

Although high yields of SP have been achieved in room temperature suspensions of thymidine microcrystals exposed to UV light for 12 hours or longer, we see a low radical yield (requiring measurement times of $\approx 1$ hour) after about 45 minutes of UV irradiation. ${ }^{28}$ Our low yield is likely due to excitation of thymidine molecules limited to the outermost layer next to the EPR sample tube, similar to the observation made by Jian et al. in the thymidine film photoreactions. ${ }^{28}$ We suggest that the radicals produced by the "met" source are left over after on-SP pathway H-atom transfer has occurred, though how these radicals become trapped remains unclear. Similarly, the TH isotopologues produced by the "O" hydrogen source may be equivalent to the "met" source (therefore on the SP pathway) and due to trace ${ }^{1} \mathrm{H}$ impurities in thymidine- $d_{3}$. The TH radicals produced by the "EX" source form through a mechanism that is not on the suggested pathway to SP formation.

\section{Conclusions}

In previous studies of deuterium-labeled thymidine, spore photoproduct was observed to form when one thymidine molecule abstracted an $\mathrm{H}$ atom from the methyl group of an adjacent thymidine onto its C6 position on route to the final product. This $\mathrm{H}$ atom abstraction is suggested to form the $\mathrm{TCH}_{2}$ and $\mathrm{TH}$ as a radical pair intermediate. We have shown that the $\mathrm{TCH}_{2}$ and $\mathrm{TH}$ radicals both form in thymidine microcrystals exposed to UV radiation under a dry argon atmosphere. We did not observe the dipolar or exchange interactions expected for a radical pair. However, using selective isotopic labeling, we did observe several sources of the 
additional hydrogen at the $\mathrm{C} 6$ position of the TH radical isotopologues, including the methyl group of another thymidine molecule. This is consistent with the mechanism in Scheme 1 and lends support to the proposed radical nature of the intermediates. Although both radicals were observed in previous EPR studies, the experimental finding that $5 S$-SP is generated as the sole photoproduct in UV irradiated thymidine microcrystals as used here establishes the biological relevance of the study to the SP photochemistry for the first time.

\section{Materials and Methods}

\section{Sample Preparation}

All chemicals were used without further purification after purchase. Gaseous nitrogen and argon were passed through a desiccant drying line before use in sample preparation. Glassware was dried in an oven overnight before use. Water vapor was avoided in sample preparation as relative humidity has been reported to influence the radicals produced in irradiated films of thymine and DNA. ${ }^{22,50}$ To prepare samples of thymidine in Figure 1, microcrystals of thymidine (Sigma-Aldrich) were suspended in diethyl ether (99\%, Sigma-Aldrich) and pipetted to the bottom of a $4 \mathrm{~mm}$ O.D. quartz EPR sample tube. The ether was removed on a vacuum manifold for a minimum of 5 hours. The EPR tube was pump-purged for three cycles under vacuum and argon and flame sealed under a lowpressure argon environment with a propane oxygen torch. A similar procedure was used for thymidine- $d_{3}$. Thymidine- $d_{3}$ was synthesized as previously described. ${ }^{26}$

To prepare H/D-thy, thymidine was dissolved in methanol- $d_{4}$ (Cambridge Isotopes, $99.8 \%$ D) to a ratio of $\geq 300: 1$ exchangeable deuterons:protons in an EPR sample tube. To prevent exposure to atmospheric water, the sample tube was stored in a desiccated environment under a constant flow of nitrogen for 24 hours, after which the methanol- $d_{4}$ was pumped off on a vacuum manifold with tip-off manifold adapter. This procedure was repeated two more times. On the final exchange, the methanol- $d_{4}$ was allowed to slowly evaporate over the course of 1-2 days by directing dry nitrogen into the EPR tube with a syringe needle. This produced powdery white crystals of H/D-thy. The exchange in methanol- $d_{4}$ appears complete as indicated by ${ }^{1} \mathrm{H}$ NMR (Figure S8). The same procedure was used to prepare $\mathrm{H} / \mathrm{D}-\mathrm{thy}-d_{3}$ from thymidine- $d_{3}$.

\section{Radical generation}

Paramagnetic signals were generated using the 266 $\mathrm{nm}$ wavelength of an $\mathrm{Nd}$ :YAG laser (Continuum, Surelite EX). Laser pulses (less than $800 \mu \mathrm{J}$ per pulse, 3-5 ns pulse length, $10 \mathrm{~Hz}$ repetition rate, $10 \mathrm{~mm}$ spot size; $10 \mathrm{~mW} / \mathrm{cm}^{2}$ ) were passed through space into a 2 $\mathrm{mm}$ aperture on a Bruker SHQE cavity EPR resonator held at the desired temperature. Low-power laser pulses make biphotonic processes unlikely in this system. ${ }^{51}$ Samples were irradiated for 5 minutes, then the sample tube was rotated to increase sample exposure to UV radiation. This was done up to a total irradiation time of 45 minutes. Samples in Figure 1A were irradiated at $120 \mathrm{~K}$. The radiation was then removed and EPR spectra collected. The samples in Figure 1B (1C) were irradiated at $210 \mathrm{~K}(250 \mathrm{~K})$ then radiation was removed. The cavity was then cooled to $120 \mathrm{~K}$ and allowed to equilibrate for 5 minutes followed by collection of EPR spectra without any additional UV radiation.

\section{EPR spectroscopy}

EPR experiments were carried out on a Bruker EMX spectrometer in an SHQE cavity resonator equipped with a variable-temperature insert. Experimental temperatures of $120 \mathrm{~K}, 210 \mathrm{~K}, 250 \mathrm{~K}$, and $260 \mathrm{~K}$ were measured on a thermocouple $\approx 2 \mathrm{~cm}$ below the sample. The desired temperature was reached with a temperature controller utilizing the flow of evaporated nitrogen through a quartz transfer line as coolant. Spectra were collected at a frequency of $\approx 9.3 \mathrm{GHz}$ (specified in figure captions) with the microwave power set to prevent saturation (see figure captions for more detail). Additional spectrometer parameters for all spectra collected were field modulation with $0.2 \mathrm{mT}$ peak-to-peak amplitude and $100 \mathrm{kHz}$ frequency, time constant of $40.96 \mathrm{~ms}$, and sweep rate of $\approx 0.7 \mathrm{mT} / \mathrm{s}$.

UV irradiation of the samples produced a sharp feature at the center of the EPR spectra attributed to radicals generated in the quartz tubes. The central 25 or fewer points were removed in the spectra plotted in Figure 1 for clarity. This central feature can be seen in the data presented in the Supplemental Information. In all cases, the spectra presented are normalized to their most intense feature (in some cases the quartz feature, SI Figures S2 and S3) for clarity.

\section{Density Functional Theory (DFT) calculations}

Geometry optimization calculations were carried out on the Gaussian09 software $^{52}$ with spin unrestricted orbitals, the B3LYP functional, and the 6-311G basis set for the $\mathrm{TCH}_{2}$ and $\mathrm{TH}$ radicals. Using the output geometry of the optimized $\mathrm{TH}$ radical, geometry optimization dependent on the methyl group rotation was carried out at the same level of theory. This was done by simultaneously incrementing the three dihedral angles from C6-C5-C7-H1, C6-C5-C7-H2, and C6-C5$\mathrm{C} 7-\mathrm{H} 3$, freezing the dihedral angles and performing a geometry optimization on the remaining structure. This was performed in 10 degree increments for 11 steps generating an output file of 12 optimized structures. The magnetic properties including g-tensors and hyperfine coupling constants were calculated using coupled-perturbed Kohn-Sham approach for the $\mathrm{TCH}_{2}$ geometry and each of the $12 \mathrm{TH}$ optimized geometries in ORCA 3.0 with spin unrestricted orbitals, the B3LYP functional, and the $6-311 \mathrm{G}$ basis set. ${ }^{53-56}$ 
Calculated Mulliken spin populations are visualized with the Chimera software. ${ }^{57}$

Spectral simulations

EPR spectra were simulated with the EasySpin software $^{58}$ using the model spin Hamiltonian

$$
\widehat{H}=\mu_{\mathrm{B}} \boldsymbol{B} \boldsymbol{g} \widehat{\boldsymbol{S}}+\sum_{k} h \widehat{\boldsymbol{S}} \boldsymbol{A}_{k} \widehat{\boldsymbol{I}}_{k}
$$

where the first term is the electron Zeeman interaction containing the Bohr magneton, $\mu_{\mathrm{B}}$, the externally applied magnetic field $\boldsymbol{B}$, the electron spin operator $\widehat{\boldsymbol{S}}$, and the electron $\mathrm{g}$ tensor, $\boldsymbol{g}$. The second term describes the hyperfine interaction between an electron spin, $\widehat{\boldsymbol{S}}$, and a nuclear spin, $\widehat{\boldsymbol{I}}_{k}$, with the hyperfine matrix $\boldsymbol{A}_{k} . k$ sums over all coupled nuclei. All tensor orientations used in the simulation were taken from the DFT calculations. The tensor orientation for each of the three methyl group hydrons in the TH isotopologues is the average orientation of the three hydrons further averaged over all twelve optimized structures. Hyperfine principal values and $g$ principal values were varied manually to the values listed in Tables 1 and 2 for $\mathrm{TCH}_{2}$ and $\mathrm{TH}$ simulations. The hyperfine coupling principal values were adjusted for deuterium nuclei (described in Results section and summarized in SI Section 1) for simulation of isotopologues.

\section{Associated Content}

Supporting Information. Full tables of magnetic properties used in EPR simulations, unrestrictive model for spectral simulations, EPR spectra after changing temperature, supplemental quantum chemical calculations, nuclear magnetic resonance spectra. This material is available free of charge via the Internet at http://pubs.acs.org.

\section{Author Information}

\section{Corresponding Author}

*(S.S.) E-mail:stst@uw.edu; phone: 206-543-2906

\section{Present Address:}

$\S$ School of Chemistry \& Chemical Engineering, Shaanxi Normal University (SNNU), No. 620, West Chang'an Avenue, Xi'an, Shaanxi, 710119, P. R. China

\section{Notes}

The authors declare no competing financial interest.

\section{Acknowledgements}

This work was in part funded by NSF grant CHE1452967 (S.S.), CHE-1454184 (L.L.), NIH training grant GM008268 (E.C.H.), and the University of Washington.

\section{References}

(1) Kneuttinger, A. C.; Kashiwazaki, G.; Prill, S.; Heil, K.; Müller, M.; Carell, T. Formation and Direct Repair of UV-Induced Dimeric DNA Pyrimidine Lesions. Photochem. Photobiol. 2014, 90, 1-14.

(2) Donnellan, J. E.; Setlow, R. B. Thymine Photoproducts but Not Thymine Dimers Found in Ultraviolet-Irradiated Bacterial Spores. Science 1965, 149, 308-310.

(3) Varghese, A. J.; Wang, S. Y. Ultraviolet Irradiation of DNA in Vitro and in Vivo Produces a Third Thymine-Derived Product. Science 1967, 156, 955-957.

(4) Kim, S. J.; Lester, C.; Begley, T. P. Synthesis of the Dinucleotide Spore Photoproduct. J. Org. Chem. 1995, 60, 6256-6257.

(5) Desnous, C.; Guillaume, D.; Clivio, P. Spore Photoproduct: A Key to Bacterial Eternal Life. Chem. Rev. 2010, 110, 1213-1232.

(6) Setlow, P.; Li, L. Photochemistry and Photobiology of the Spore Photoproduct: A 50Year Journey. Photochem. Photobiol. 2015, 91, 1263-1290.

(7) Buis, J. M.; Cheek, J.; Kalliri, E.; Broderick, J. B. Characterization of an Active Spore Photoproduct Lyase, a DNA Repair Enzyme in the Radical S -Adenosylmethionine Superfamily. J. Biol. Chem. 2006, 281, 2599426003.

(8) Chandor-Proust, A.; Berteau, O.; Douki, T.; Gasparutto, D.; Ollagnier-de-Choudens, S.; Fontecave, M.; Atta, M. DNA Repair and Free Radicals, New Insights into the Mechanism of Spore Photoproduct Lyase Revealed by Single Amino Acid Substitution. J. Biol. Chem. 2008, 283, 36361-36368.

(9) Chandra, T.; Silver, S. C.; Zilinskas, E.; Shepard, E. M.; Broderick, W. E.; Broderick, J. B. Spore Photoproduct Lyase Catalyzes Specific Repair of the 5R but Not the 5S Spore Photoproduct. J. Am. Chem. Soc. 2009, 131, 2420-2421.

(10) Benjdia, A.; Heil, K.; Barends, T. R. M.; Carell, T.; Schlichting, I. Structural Insights into Recognition and Repair of UV-DNA Damage by Spore Photoproduct Lyase, a Radical SAM Enzyme. Nucleic Acids Res. 2012, 40, 9308 9318.

(11) Kneuttinger, A. C.; Heil, K.; Kashiwazaki, G.; Carell, T. The Radical SAM Enzyme Spore Photoproduct Lyase Employs a Tyrosyl Radical for DNA Repair. Chem. Commun. 2013, 49, 722-724.

(12) Yang, L.; Nelson, R. S.; Benjdia, A.; Lin, G.; Telser, J.; Stoll, S.; Schlichting, I.; Li, L. A Radical Transfer Pathway in Spore Photoproduct Lyase. Biochemistry 2013, 52, 3041-3050.

(13) Yang, L.; Li, L. Spore Photoproduct Lyase: The 
Known, the Controversial, and the Unknown. $J$. Biol. Chem. 2015, 290, 4003-4009.

(14) Cano, R. J.; Borucki, M. K. Revival and Identification of Bacterial Spores in 25- to 40Million-Year-Old Dominican Amber. Science 1995, 268, 1060-1064.

(15) Setlow, P. Spores of Bacillus Subtilis : Their Resistance to and Killing by Radiation, Heat and Chemicals. J. Appl. Microbiol. 2006, 101, 514525 .

(16) Marshall, M. M.; Hayes, S.; Moffett, J.; Sterling, C. R.; Nicholson, W. L. Comparison of UV Inactivation of Spores of Three Encephalitozoon Species with That of Spores of Two DNA Repair-Deficient Bacillus Subtilis Biodosimetry Strains. Appl. Environ. Microbiol. 2003, 69, 683-685.

(17) Nicholson, W. L.; Galeano, B. UV Resistance of Bacillus Anthracis Spores Revisited: Validation of Bacillus Subtilis Spores as UV Surrogates for Spores of B. Anthracis Sterne. Appl. Environ. Microbiol. 2003, 69, 1327-1330.

(18) Guerrero-Beltràn, J. A.; Barbosa-Cànovas, G. V. Review : Advantages and Limitations on Processing Foods by UV Light. Food Sci. Technol. Int. 2004, 10, 137-147.

(19) Rahn, R. O.; Hosszu, J. L. Photoproduct Formation in DNA at Low Temperatures. Photochem. Photobiol. 1968, 8, 53-63.

(20) Varghese, A. J. 5-Thyminyl-5,6Dihydrothymine from DNA Irradiated with Ultraviolet Light. Biochem. Biophys. Res. Commun. 1970, 38, 484-490.

(21) Pruden, B.; Snipes, W.; Gordy, W. Electron Spin Resonance of an Irradiated Single Crystal of Thymidine. Proc. Natl. Acad. Sci. 1965, 53, 917-924.

(22) Pershan, P. S.; Shulman, R. G.; Wyluda, B. J.; Eisinger, J. Electron Spin Resonance of Irradiated DNA. Science 1965, 148, 378-380.

(23) Varghese, A. J. Photochemistry of Thymidine in Ice. Biochemistry 1970, 9, 4781-4787.

(24) Cadet, J.; Vigny, P. The Photochemistry of Nucleic Acids. In Bioorganic Photochemistry: Photochemistry and the Nucleic Acids; Morrison, H., Ed.; J. Wiley \& Sons: New York, 1990; Vol. 1, pp 96-99.

(25) Lin, G.; Li, L. Elucidation of SporePhotoproduct Formation by Isotope Labeling. Angew. Chem. Int. Ed. 2010, 49, 9926-9929.

(26) Ames, D. M.; Lin, G.; Jian, Y.; Cadet, J.; Li, L. Unusually Large Deuterium Discrimination during Spore Photoproduct Formation. J. Org. Chem. 2014, 79, 4843-4851.

(27) Young, D. W.; Tollin, P.; Wilson, H. R. The Crystal and Molecular Structure of Thymidine. Acta Crystallogr. Sect. B Struct. Crystallogr. Cryst. Chem. 1969, 25, 1423-1432. Jian, Y.; Ames, D. M.; Ouyang, H.; Li, L. Photochemical Reactions of Microcrystalline Thymidine. Org. Lett. 2015, 17, 824-827. Herak, J. N.; Galogaza, V. Radical
Transformation in Irradiated DNA and Its Constituents. Proc. Natl. Acad. Sci. U. S. A. 1969, 64, 8-12.

(30) Box, H. C.; Budzinski, E. E.; Potter, W. R. ENDOR Characterization of the Free Radical in Irradiated Thymidine. J. Chem. Phys. 1974, 61, 1136-1139.

(31) Herak, J. N.; McDowell, C. A. ENDOR Study of the Minority Radicals Formed in X-RayIrradiated Single Crystals of Thymidine. $J$. Magn. Reson. 1974, 16, 434-438.

Hole, E. O.; Sagstuen, E.; Nelson, W. H.; Close, D. M. Primary Reduction and Oxidation of Thymine Derivatives. ESR/ENDOR of Thymidine and 1-Methylthymine X-Irradiated at 10 K. J. Phys. Chem. 1991, 95, 1494-1503.

(33) Sagstuen, E.; Hole, E. O.; Nelson, W. H.; Close, D. M. Radiation-Induced Free-Radical Formation in Thymine Derivatives. EPR/ENDOR of Anhydrous Thymine Single Crystals X-Irradiated at $10 \mathrm{~K}$. J. Phys. Chem. 1992, 96, 1121-1126.

(34) Flossmann, W.; Zehner, H.; Müller, A. Radicals Produced in Single Crystals of Thymine Derivatives by UV and X-Rays. Z. Naturforsch. 1980, 35, 20-29.

(35) Herak, J. N. EPR Study of Radiation Damage of Deuterated Single Crystal of Thymidine. J. Chem. Phys. 1970, 52, 6440-6441.

(36) Dulcic, A.; Herak, J. N. Radiation-Induced PairWise Radical Formation in Single Crystals of Thymine. Biochim. Biophys. Acta 1973, 319, 109-115.

(37) Holroyd, R. A.; Glass, J. W. Radicals Formed by Electron Transfer to Pyrimidine Derivatives. Int. J. Radiat. Biol. 1968, 14, 445-452.

(38) Sevilla, M. D. An Electron Spin Resonance Study of the Photoionization of Thymine. The Thymine Cation and Anion Radicals. J. Phys. Chem. 1971, 75, 626-631.

(39) Sevilla, M. D.; Van Paemel, C.; Nichols, C. An Electron Spin Resonance Study of Several Base Cation Radicals Produced by Photoionization. $J$. Phys. Chem. 1972, 76, 3571-3577.

(40) Sevilla, M. D.; Van Paemel, C.; Zorman, G. Reactions of the Cation and Anion Radicals of Several DNA Bases. J. Phys. Chem. 1972, 76, 3577-3582.

(41) Adhikary, A.; Kumar, A.; Heizer, A. N.; Palmer, B. J.; Pottiboyina, V.; Liang, Y.; Wnuk, S. F.; Sevilla, M. D. Hydroxyl Ion Addition to OneElectron Oxidized Thymine: Unimolecular Interconversion of $\mathrm{C} 5$ to $\mathrm{C} 6 \mathrm{OH}$-Adducts. $\mathrm{J}$. Am. Chem. Soc. 2013, 135, 3121-3135.

(42) Deeble, D. J.; Das, S.; von Sonntag, C. Uracil Derivatives: Sites and Kinetics of Protonation of the Radical Anions and the UV Spectra of the $\mathrm{C}(5)$ and C(6) H-Atom Adducts. J. Phys. Chem. 1985, 89, 5784-5788.

(43) Novais, H. M.; Steenken, S. ESR Studies of Electron and Hydrogen Adducts of Thymine and Uracil and Their Derivatives and of 4,6- 
Dihydroxypyrimidines in Aqueous Solution. Comparison with Data from Solid State. The Protonation at Carbon of the Electron Adducts. J. Am. Chem. Soc. 1986, 108, 1-6.

(44) Box, H. C.; Budzinski, E. E. Primary Radiation Damage in Thymidine. J. Chem. Phys. 1975, 62, 197-199.

(45) Sagstuen, E.; Hole, E. O.; Nelson, W. H.; Close, D. M. Structure of the Primary Reduction Product of Thymidine after X Irradiation at 10 K. J. Phys. Chem. 1989, 93, 5974-5977.

(46) Cullis, P. M.; Evans, P.; Malone, M. E. Electron Addition to DNA-Thymine vs Cytosine Radical Anion? Chem. Commun. 1996, 985-986.

(47) Adhikary, A.; Kumar, A.; Becker, D.; Sevilla, M. D. Understanding DNA Radicals Employing Theory and Electron Spin Resonance Spectroscopy. Encyclopedia of Radicals in Chemistry, Biology and Materials, 2012, 1-26.

(48) Malone, M. E.; Symons, M. C. R.; Parker, A. W. An EPR Study of Photoionised Thymine and Its Derivatives at 77 K. J. Chem. Soc. Perkin Trans. 2 1993, 2067-2075.

(49) Du, Q.; Zhao, H.; Song, D.; Liu, K.; Su, H. Consecutive Reaction Mechanism for the Formation of Spore Photoproduct in DNA Photolesion. J. Phys. Chem. B 2012, 116, 11117-11123.

(50) Rahn, R. O.; Hosszu, J. L. Influence of Relative Humidity on the Photochemistry of DNA Films. Biochim. Biophys. Acta 1969, 190, 126-131.

(51) Angelov, D.; Berger, M.; Cadet, J.; Getoff, N.;
Keskinova, E.; Solar, S. Comparison of the Effects of High-Power U.V.-Laser Pulses and Ionizing Radiation on Nucleic Acids and Related Compounds. Internatial J. Radiat. Appl. Instrum. Part C 1991, 37, 717-727.

(52) Gaussian 09, Revision C.01, Frisch, M. J.; Trucks, G. W.; Schlegel, H. B.; Scuseria, G. E.; Robb, M. A.; Cheeseman, J. R.; Scalmani, G.; Barone, V.; Mennucci, B.; Petersson, G. A.; et al. Gaussian, Inc., Wallingford CT, 2010.

(53) Neese, F. The ORCA Program System. WIRES: Comput. Mol. Sci., 2012, 2, 73-78.

(54) Lee, C.; Yang, W.; Parr, R. G. Development of the Colle-Salvetti Correlation-Energy Formula into a Functional of the Electron Density. Phys. Rev. B 1988, 37, 785-789.

(55) Becke, A. D. A New Mixing of Hartree-Fock and Local Density-Functional Theories. $J$. Chem. Phys. 1993, 98, 1372-1377.

(56) Krishnan, R.; Binkley, J. S.; Seeger, R.; Pople, J. A. Self-Consistent Molecular Orbital Methods. XX. A Basis Set for Correlated Wave Functions. J. Chem. Phys. 1980, 72, 650-654.

(57) Pettersen, E. F.; Goddard, T. D.; Huang, C. C.; Couch, G. S.; Greenblatt, D. M.; Meng, E. C.; Ferrin, T. E. UCSF Chimera--A Visualization System for Exploratory Research and Analysis. J. Comput. Chem. 2004, 25, 1605-1612.

(58) Stoll, S.; Schweiger, A. EasySpin, a Comprehensive Software Package for Spectral Simulation and Analysis in EPR. J. Magn. Reson. 2006, 178, 42-55. 
TOC Figure

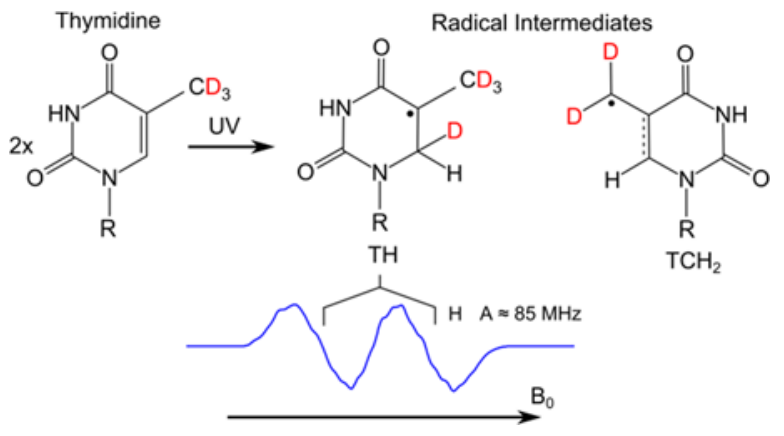

